\title{
ACEN Position Statement: Nursing Workload-A Priority for Healthcare
}

\author{
Mary Ferguson-Paré, RN, PhD \\ Vice President, Professional Affairs, and Chief Nurse Executive \\ University Health Network \\ Toronto, ON
}

The Academy of Canadian Executive Nurses (ACEN), the organization of chief nurse executives of teaching hospitals across Canada, has agreed on this position statement on nursing workload. In a previous issue of the Canadian Journal of Nursing Leadership, Affonso et al. (2003) identified workload as a significant issue for nurses in delivering processes of care in a way that supports patient safety. We cannot underestimate the importance of appropriate work design and nursing workload to ensure patient safety and the retention of an adequate number of nurses.

We hope that you will find this position statement a useful tool in planning for the future.

\section{ACEN Position}

The Academy of Canadian Executive Nurses believes nursing workload is one of the key critical issues to be addressed to ensure patient safety, retention of an adequate number of nurses for Canada and the ability of nurses in Academic Health Sciences Corporation (AHSC) centres to fulfill their mandate for care, teaching, research and innovation.

\section{Current Situation in Workplace}

Currently, nurses perceive that workload exceeds current staffing levels and the capacity of nurses. Nurses are unable to complete all nursing care to the standards defined by professional regulatory bodies. The continued focus on cost and efficiency does not acknowledge the increasing complexity of care being delivered and the impact of cost-saving measures on quality of care. The negative health outcomes arising from excessive workload for the nursing community and the difficulties of retaining students and employees in nursing are compelling reasons to address the negative influence of onerous workload. 


\section{Strategies for the Future}

A significant body of research evidence links adequate nurse staffing to positive patient outcomes and identifies ways to improve the nursing workplace and provide reasonable workload for nurses. Nursing leaders and healthcare executives must embrace their accountability to design workload in accordance with patient care needs and enable nurses to work to their full scope of practice. The following strategies are suggested:

- Create quality work environments/workplaces.

Identify reasonable work assignments.

- Provide appropriate equipment/tools to carry out nursing work.

- Provide adequate staff to perform care, support activities.

- Identify and provide an appropriate mix of professional staff.

- Provide development and education opportunities for nursing staff.

- Fiscal planning and nursing budgets should provide for:

additional resources in terms of time and active preceptorship/supervision to allow for appropriate education/orientation for new graduates and students

- adequate replacement staff for those who are ill, on education time or on leave $w w w . k g h . \circ n \cdot c a$

\section{A Thousand Ways to Enjoy Life!}

KINGSTON GENERAL HOSPITAL, a 445-bed academic health sciences centre affiliated with Queen's University and a Cancer Care Ontario partner, provides tertiary care to the 500,000 people of Southeastern Ontario. We are mid-way between Toronto and Montreal at the gateway to the St. Lawrence River and the 1000 Islands. History, culture, recreation, entertainment, and a rich academic community combine to make Kingston a showcase for quality living .. .

Consider KGH for a career opportunity as:
- RN's

- Educators

- Managers

- Directors
- Administrative Coordinators

- Advance Practice Nurses
For a thousand ways to enjoy life and work come to Kingston! We offer a proactive, results-oriented, and professional practice environment where innovation is rewarded.

Visit our website at www.kgh.on.ca for details about these and other exciting career opportunities.

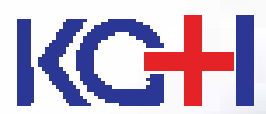

KINGSTON GENERAL HOSPITAL

A National Historic Site.

- adjusting productivity expectations through acknowledgment of such variables as age and experience of the nursing workforce

- Invest in nursing leadership development to lead and sustain the profession.

- Enable nursing staff working in partnership with other health professionals to live their full scope of practice.

- Focus on work design and an environment that acknowledges the needs of both care providers and patients.

- Invest in development of new tools and methodologies to predict, measure and validate nursing workload. 
- Determine appropriate nursing productivity benchmarks that include measures of quality of care and patient outcomes as well as the health and performance of the nursing workforce.

\section{Indicators of Success}

In order to meet the needs of patients/families while ensuring appropriate nursing workloads, the following should be monitored:

- delivered hours of care per day/visits per specialty area

- utilization of full-time, part-time, casual and per diem staff

- utilization of paid nursing hours:

- overtime

- education

- sick time

- staff outcomes to include satisfaction, workplace injury, sick leave, etc.

- nursing retention and turnover rates

- amount of funding spent on nursing equipment and other tools

- monitoring of nurse-sensitive patient outcomes

- patient satisfaction with nursing care

- ratio of nurses to population

\section{References}

Affonso, D.D., L. Jeffs, D. Doran and M. Ferguson-Paré. 2003. "Patient Safety to Frame and Reconcile Nursing Issues." Canadian Journal of Nursing Leadership 16(4): 69-81.

Aiken, L.H., S.P. Clarke, D.M. Sloane, R. Sochalski, H. Busse, P. Clarke, J. Giovannetti, J. Hunt, A.M. Rafferty and J. Shamian. 2001. "Nurses' Reports on Hospital Care in Five Countries." Health Affairs 20(3): 43-53.

Baumann, A., L. O'Brien-Pallas, M. ArmstrongStassen, J. Blythe, R. Bourbonnais, S. Cameron, D. Irvine Doran, M. Kerr, L. McGillis Hall, M. Zina, M. Butt and L. Ryan. 2001. Commitment and Care: The Benefits of a Healthy Workplace for

Nurses, Their Patients and the System. Report submitted to the Canadian Health Services Research Foundation, Ottawa, ON.

Canadian Nursing Advisory Committee (CNAC). 2002. Our Health, Our Future: Creating Quality Workplaces for Canadian Nurses. Ottawa: Advisory Committee on Health Human Resources.

Fagin, C.M. 2001. When Care Becomes a Burden: Diminishing Access to Adequate Nursing. Monograph, The Millbank Fund.

Ferguson-Paré, M.L., G.J. Mitchell, K. Perkin and L. Stevenson. 2002 (Sept./Oct.). "ACEN Background Paper on Leadership." Canadian Journal of Nursing Leadership 15(3): 4-8.

McGillis Hall, L., D.I. Doran, G.R. Baker, G.H. Pink, S. Sidani, L. O'Brien-Pallas and G.J. Donner. 2001. A Study of the Impact of Nursing Staff Mix Models and Organizational Change Strategies on Patient, System and Nurse. Toronto: Faculty of Nursing, University of Toronto and Canadian Health Services Research Foundation/Ontario Council of Teaching Hospitals. ISBN 0-7727-3603-0.

Needleman, J., P. Buerhaus, S. Mattke, M. Stewart and K. Zelevinsky. 2002. "Nurse Staffing Levels and the Quality of Care in Hospitals." New England Journal of Medicine 346(22): 1715-22.

Nicklin, W. and J.E. McVeety. 2002. "Canadian Nurses' Perception of Patient Safety in Teaching Hospitals: Wake Up Call!" Canadian Journal of Nursing Leadership 15(3): 11-21.

Shamian, J., L. O'Brien-Pallas, M. Kerr and M. Koehoorn. 2001. Effects of Job Strain, Hospital Organizational Factors and Individual

Characteristics of Work-Related Disability among Nurses. Toronto: Ontario Workplace Safety and Insurance Board.

Tourangeau, A.E., P. Giovanetti, J.V. Vu and M. Wood. 2002. "Nursing Related Determinants of 30 Day Mortality for Hospitalized Patients." Canadian Journal of Nursing Research 33(4): 71-88.

Prescription drugs on a high New data from IMS Health finds that spending on prescription drugs in the United States and Canada increased $11 \%$ last year to $\$ 229.5$ billion, making up close to half of all worldwide sales. Spending in EU member states grew a "solid" $8 \%$ to $\$ 115.4$ billion, while sales in the rest of Europe increased $14 \%$ to reach $\$ 14.3$ billion. (Source: Washington Post, March 16, 2004) 